\title{
Upregulation of Reg $1 \alpha$ and GW112 in the epithelium of inflamed colonic mucosa
}

\author{
S Shinozaki, T Nakamura, M Iimura, Y Kato, B Iizuka, M Kobayashi, N Hayashi
}

\begin{abstract}
Background and aims-Colonic epithelium is involved in the regulation of intestinal function and mucosal immune responses, and its function is altered in inflammatory bowel disease (IBD). However, a comprehensive analysis of the genetic alterations in inflamed colonic epithelium is not available at present. The aim of our study was to detect genes that are preferentially expressed in inflamed colonic epithelia and clarify the biochemical responses of epithelial cells in inflamed colonic mucosa.
\end{abstract}

Methods-cDNA representation difference analysis was used to identify candidate genes selectively expressed in inflamed colonic epithelia. Selective expression of these genes in the epithelium of inflamed colonic mucosa, including IBD and non-IBD tissues, was examined by real time polymerase chain reaction and in situ hybridisation. The effect of cell confluence and inflammatory mediators on Reg $1 \alpha$ gene expression was examined using a colon cancer cell line (HT29).

Results-We identified seven candidate genes that were presumed to be upregulated in the inflamed colonic epithelium. Of these, Reg $1 \alpha$ and GW112 were the dominant species and expression of these genes was confined to the crypt epithelium. In vitro studies using a colonic epithelial cell line suggested that cell confluence regulates Reg $1 \alpha$ gene expression. Conclusions-Selective expression of Reg $1 \alpha$ and GW112 genes in the crypt epithelium of inflamed colonic mucosa suggests the important regulatory functions of these genes.

(Gut 2001;48:623-629)

Keywords: Reg 1 $\alpha$; GW112; Annexin-1; inflammatory bowel disease; representation difference analysis

The colonic epithelium provides a barrier against potentially injurious luminal agents such as acids, enzymes, bacteria, and toxins, and is known to play an active role in the immune response of the intestinal mucosa. A breakdown of colonic epithelium function is thought to be the salient feature of a variety of common and important gastrointestinal disorders, including inflammatory bowel disease (IBD). ${ }^{12}$ Several general and specific protective factors are thought to be involved in the maintenance of epithelial function. ${ }^{2}$ Colitis in knockout mice lacking interleukin (IL) $-2{ }^{3}$
IL-10, ${ }^{4}$ or T cell receptor alpha, ${ }^{5}$ and in dominant negative $\mathrm{N}$-cadherin transgenic mice ${ }^{6}$ is thought to result from changes in either the mucosal immune system or the epithelial cell-cell adhesion system, or both. There is ample evidence that alteration in colonic epithelial cell phenotype and function is present in IBD. For example, epithelial permeability is increased in IBD, ${ }^{7}$ and epithelial proliferation is more rapid in IBD than in normal subjects. ${ }^{8}$ Furthermore, there is altered antigen presentation by epithelial cells in IBD. ${ }^{910}$ Intestinal epithelial cells not only express a variety of immune receptors ${ }^{11}$ but can also produce a wide array of immunomodulatory substances such as IL-1 $\beta,{ }^{12}$ IL- $6,{ }^{12}$ IL- $8,{ }^{13}$ C-X-C chemokine ENA-78, ${ }^{14}$ IL-15, ${ }^{15}$ and inducible nitric oxide synthase. ${ }^{16}$ These results imply a central role for colonic epithelial cells in the regulation of intestinal function and mucosal immune responses and prompted us to further examine alterations of colonic epithelial cells in inflamed colonic mucosa. For this purpose, we used the representation difference analysis (RDA) method to detect genes that are differentially expressed in the colonic epithelium in inflamed colonic mucosa.

\section{Patients and methods}

PATIENTS AND TISSUES

Colonic biopsy specimens were obtained by endoscopy from 21 patients with ulcerative colitis (UC) (in three patients colonic biopsy specimens were obtained at different time points during the active and inactive phases of the disease), from nine patients with Crohn's disease, five patients with ischaemic colitis, one patient with amoebic colitis, and six normal controls (table 1). The diagnosis of UC and Crohn's disease was based on established endoscopic and histological criteria. ${ }^{17}$ The diagnosis of ischaemic colitis was based on clinical features and established endoscopic criteria, ${ }^{18}$ while that of amoebic colitis was based on the presence of the organism. Inactive UC represented cases with endoscopically healed areas and tissue specimens featuring crypt atrophy or distortion with no neutrophil infiltration. On the other hand, active UC

Abbreviations used in this paper: $\mathrm{AP}$, alkaline phosphatase; GAPDH, glyceraldehyde-3-phosphate dehydrogenase; hr, human recombinant; IBD, inflammatory bowel disease; IL, interleukin; IFN- $\gamma$, interferon $\gamma$; TNF- $\alpha$, tumour necrosis factor $\alpha$; SIN-1, 3-morpholinosydnonimine; PCR, polymerase chain reaction; DP, difference product; Dig, digoxigenin; ECL, enterochromaffin; fg, femtogram; RDA, representation difference analysis; Reg $1 \alpha$ R, Reg $1 \alpha$ receptor; UC, ulcerative colitis; ISH, in situ hybridisation. 
Table 1 Demographic features of the participating patients

\begin{tabular}{|c|c|c|c|c|c|c|}
\hline \multirow[b]{2}{*}{ Mucosal disease activity } & \multicolumn{2}{|l|}{$U C$} & \multicolumn{2}{|l|}{$C D$} & \multicolumn{2}{|c|}{ Non-IBD } \\
\hline & Active & Inactive & Active & Inactive & Active & Control \\
\hline $\mathrm{n}$ & 14 & 10 & 4 & 5 & 6 & 6 \\
\hline $\operatorname{Sex}(M / F)$ & $8 / 6$ & $7 / 3$ & $3 / 1$ & $4 / 1$ & $1 / 4$ & $4 / 2$ \\
\hline \multicolumn{7}{|l|}{ Age $(y)$} \\
\hline Range & $24-59$ & $33-65$ & $19-44$ & $19-52$ & $44-64$ & $32-72$ \\
\hline Mean & 38.2 & 38.7 & 28.2 & 30.4 & 52.6 & 53.3 \\
\hline \multicolumn{7}{|l|}{ Disease duration (y) } \\
\hline Range & $1-19$ & $3-18$ & $2-8$ & $2-20$ & & \\
\hline Mean & 8.3 & 10.2 & 5.0 & 10.4 & & \\
\hline \multicolumn{7}{|l|}{ Treatment } \\
\hline Cyclosporin & 2 & 0 & 0 & 0 & & \\
\hline Steroid & 4 & 4 & 2 & 2 & & \\
\hline $\begin{array}{l}\text { Sulphasalazine/5-ASA } \\
\text { only }\end{array}$ & $4 / 1$ & $5 / 0$ & $0 / 1$ & $0 / 2$ & & \\
\hline No treatment & 3 & 1 & 1 & 1 & & \\
\hline
\end{tabular}

UC, ulcerative colitis; CD, Crohn's disease; IBD, inflammatory bowel disease.

included cases with endoscopically inflamed areas of low to moderate grade (increased granularity and friability of the mucosa) but areas of erosion and ulceration were excluded from analysis. Histological inflammatory activity was evaluated based on the degree of neutrophil infiltration according to Matts' grade $^{19}$ : grades 1 and 2 were classified as active in this study. Informed consent was obtained from all patients and control subjects (patients with colorectal cancer and polyps) before biopsy. The study protocol was approved by the Institutional Review Committee for Research on Human Subjects. Demographic features of the patients and history of drug therapy up to the time of colonoscopy are summarised in table 1 .

CELL LINE, CYTOKINES, AND REAGENTS

The human colon cancer cell line (HT29) was obtained from the American Type Culture Collection (Rockville, Maryland, USA). Recombinant human (rh) IL-1 $\beta$, rhIL-6, interferon $\gamma(\operatorname{rhIFN}-\gamma)$, and tumour necrosis factor $\alpha$ (rhTNF- $\alpha$ ) were purchased from Biosource (Camarillo, California, USA). rhIL-4 was purchased from PharMingen (San Diego, California, USA) and 3-morpholinosydnonimine (SIN-1) from Sigma (St Louis, Missouri, USA).

ISOLATION OF COLONIC CRYPT AND SURFACE EPITHELIUM

Colonic crypts and surface epithelia were isolated from colonic biopsy specimens in sheets consisting of both crypt and surface epithelium using the method previously described by our laboratory. ${ }^{20}$ Briefly, immediately after obtaining the colonic biopsy, the specimen was immersed in $10 \mathrm{ml}$ of $\mathrm{Ca}^{2+}$ and $\mathrm{Mg}^{2+}$ free Hank's balanced salt solution (Life Technologies, Gaithersburg, Maryland, USA) containing $30 \mathrm{mM}$ EDTA and incubated at room temperature for 30 minutes. After incubation the colonic crypts and surface epithelia were isolated mechanically by microscopy using fine needles. The isolated epithelium was used to extract RNA. That the isolated samples contained only crypt and surface epithelium was demonstrated morphologically using haematoxylin and eosin stained sections examined under a light microscope.
CDNA REPRESENTATION DIFFERENCE ANALYSIS (RDA)

RDA for cDNA was performed as described previously. ${ }^{21}$ In brief, cDNA from normal or active UC colonic epithelium was digested with $D p n \mathrm{II}$ and ligated to the R-Bgl-12/24 adapters. Representations were made by polymerase chain reaction (PCR) amplification of the $R$ ligated cDNA fragments for 20 cycles using the $\mathrm{R}-\mathrm{Bgl}-24 \mathrm{mer}$ as primer. The $\mathrm{R}$ linked sequences in tester representations were replaced with J-Bgl-12/24 adaptors. Firstly, subtractive hybridisation was set up using $0.4 \mu \mathrm{g}$ of $\mathrm{J}$ ligated testers and $40 \mu \mathrm{g}$ of linker removed drivers (tester:driver=1:100). An aliquot of the hybridisation mixture was amplified by PCR for 10 cycles using the J-24mer as primers. The PCR products were then digested with mung bean nuclease (New England Biolabs, Beverly, Massachusetts, USA) for 35 minutes at $30^{\circ} \mathrm{C}$. Digested PCR products were further amplified for 18 cycles, and the products of this amplification were the first difference products (DP1). The procedure was repeated twice using different primers and tester:driver ratios of 1:800 and 1:80 000 .

IN SITU HYBRIDISATION (ISH)

Biopsy specimens were fixed with $20 \%$ neutral formalin immediately after harvesting. Formalin fixed materials were embedded in paraffin using standard procedures. Deparaffinised and rehydrated sections $(4 \mu \mathrm{m})$ were treated with $10 \mu \mathrm{g} / \mathrm{ml}$ of proteinase $\mathrm{K}$ at $37^{\circ} \mathrm{C}$ for 20 minutes. Hybridisation was performed overnight using $5 \mu \mathrm{g} / \mathrm{ml}$ of digoxigenin (Dig)-UTP labelled sense and antisense RNA probes at $60^{\circ} \mathrm{C}$. To produce Dig labelled RNA, cDNAs of Reg $1 \alpha$ and GW112 inserted into pCRII vector (Invitrogen, San Diego, California, USA) with both orientation was transcribed in vitro with T7 RNA polymerase in the presence of Dig-UTP using the DIG RNA labelling kit (Boehringer Mannheim, Mannheim, Germany), as explained in the protocol provided by the manufacturer. After appropriate washing with $50 \%$ formamide in $0.5 \times \mathrm{SSC}$ at $45^{\circ} \mathrm{C}$, sections were reacted with alkaline phosphatase (AP) sheep anti-Dig antibody, and the sites of AP were visualised with NBT/BCIP (Boehringer Mannheim). To verify the specificity of signals, Dig labelled sense RNA probes were hybridised with adjacent sections as negative controls in every experiment.

\section{DNA SEQUENCING}

The primer set used to amplify full length Reg $1 \alpha$ cDNA was 5'-AGCATGGCTCAGACCA GCTCATAC-3' for 5' primer and 5'-CCT CTAGTTTTTGAACTTGCAGAC-3' for 3' primer. The cycling conditions were $94^{\circ} \mathrm{C}$ for 30 seconds, $55^{\circ} \mathrm{C}$ for 30 seconds, and $68^{\circ} \mathrm{C}$ for two minutes for 35 cycles with platinum Taq DNA polymerase High Fidelity (Life Technologies). Amplified Reg $1 \alpha$ cDNA was cloned into pCRII vector (Invitrogen) followed by DNA sequencing using M13 reverse and forward primers on ALF automated DNA sequencer II (Pharmacia Biotech). 
REAL TIME QUANTITATIVE PCR WITH

FLUOROGENIC PROBES

Total RNA was extracted as previously described $^{22}$ from the isolated specimens of colonic epithelium. Reverse transcription of extracted RNA (500 ng) was performed using RNase H deficient reverse transcriptase (Superscript II; Life Technologies) and oligo(dT) primers (Life Technologies). An aliquot $(2 \mu \mathrm{l})$ of diluted reverse transcription reaction mixture $(200 \mu \mathrm{l})$ was used for quantitation of Reg $1 \alpha$, GW112, Annexin-1, and glyceraldehyde-3-phosphate dehydrogenase (GAPDH) gene expression by real time PCR assay. The primer set used to amplify Reg $1 \alpha$ was 5'-TCCCTGG TCTCCTACAAGTCCT-3' for 5' primer and 5'-GGAATCCTGTGCTTGAGGTCAG-3' for 3' primer. The primer set used to amplify GW112 was 5'-TGGTGTGGTGAAC ATCAGCA-3' for '5' primer and 5'CCTACCCCAAGCACCATATAGA-3' for 3' primer. The primer set used to amplify Annexin-1 was 5'-AGTTCTGGACCTG GAGTTGAAAG-3' for 5' primer and 5'TGCAAAGAAAGCTGGTTTGC-3' for 3' primer. The primer set used to amplify GAPDH was 5'-GAAGGTGAAGGTCGGA GTC-3' for 5' primer and 5'-GAAG ATGGTGATGGGATTTC-3' for 3' primer. The FAM conjugated fluorogenic probes used to quantify Reg $1 \alpha$, GW112, and Annexin-1 gene expression were 5'-FAM-TGGAG CCCCAAGCAGTGTTAATCCTG-3', 5'FAM-ACCGTCTGTGGTTCAGCTCAAC TGGA-3', and 5'-FAM-AGAAATGCCTCAC AGCTATCGTGAAGTGC-3', respectively. The JOE conjugated fluorogenic probe used to quantify GAPDH gene expression was 5'-JOECAAGCTTCCCGTTCTCAGCC-3'. The fluorogenic probes were synthesised by $\mathrm{PE}$ Applied Biosystems (Foster City, California, USA). PCR was performed using the Taq-Man PCR kit (PE Applied Biosystems), as previously described. ${ }^{23}$ Following activation of the AmpliTaq Gold (PE Applied Biosystems) for 10 minutes at $95^{\circ} \mathrm{C}, 35$ cycles of 15 seconds at $95^{\circ} \mathrm{C}$ and one minute at $62^{\circ} \mathrm{C}$ were carried out using a Sequence Detector (model 7700, PE Applied Biosystems). Real time fluorescence measurements were taken and a threshold cycle $\left(\mathrm{C}_{\mathrm{T}}\right)$ value for each sample was calculated by the above sequence detector. ${ }^{24}$ For Reg $1 \alpha$, GW 112, and Annexin-1, standard curves of $\mathrm{C}_{\mathrm{T}}$ values obtained from serially diluted pCRIIReg $1 \alpha$, pCRIIGW112, and pCRIIAnn-1 were constructed. $\mathrm{C}_{\mathrm{T}}$ values for Reg $1 \alpha$, GW112, and Annexin-1 transcripts from clinical specimens were plotted on the standard curves, and the amounts (fg, femtogram) of these genes were calculated automatically by Sequence Detector version 1.6 (PE Applied Biosystems). The amounts are expressed as the amount of Reg 1 $\alpha$, GW112, and Annexin-1 transcripts and not as the amount of plasmid. For GAPDH, a standard curve of $\mathrm{C}_{\mathrm{T}}$ values obtained from serially diluted standard samples was constructed. The $\mathrm{C}_{\mathrm{T}}$ values for GAPDH transcripts from clinical specimens were plotted on the standard curve and the relative amounts of GAPDH transcripts to the standard sample were calculated. Each sample was tested in duplicate, and the average of the two values was used for calculation. The difference between the two values of the samples was almost zero. After standardisation, the amount of transcripts of these genes was expressed (fg) relative to that of GAPDH. Samples were considered negative if $\mathrm{C}_{\mathrm{T}}$ values exceeded 35 cycles. Group data were expressed as mean (SEM) and differences in transcript levels between groups were analysed by the Student's $t$ test. A p value less than $5 \%$ denoted the presence of a significant difference between the tested groups.

\section{Results}

IDENTIFICATION OF CANDIDATE GENES

SELECTIVELY EXPRESSED IN NORMAL AND UC

COLONIC MUCOSA

To identify candidate genes selectively expressed in normal and UC colonic mucosa, we performed cDNA RDA between normal and active UC colonic epithelium (fig 1). Representations of normal and active UC colonic epithelium were prepared from cDNA derived from normal and active UC colonic epithelial cells. RDA was performed with the active UC representation as "tester" and an excess amount of the normal representation as "drivers" (fig 1A) or with the normal representation as "tester" and an excess amount of the active UC representation as "drivers" (fig 1B). After one round of subtraction, the first difference products (DP1) showed a broad smear band in both cases. After two rounds of subtraction, the second difference products (DP2) showed some discrete bands in both cases. After three rounds of subtraction, the third difference products (DP3) showed two discrete bands with no polydisperse DNAs in RDA with the active UC representation as "tester" and an excess amount of the normal representation as "drivers" (fig 1A). In RDA with the normal representation as "tester" and an excess amount of the active UC representation as "drivers", no discrete bands were seen (fig 1B). DP3 in RDA with the active UC representation as "tester" and the normal representation as "drivers" were randomly cloned. Of 14 clones examined, five were found to be identical to Reg $1 \alpha$, four were found to be GW112, one was zinc finger protein 17 , one was zinc finger protein 173, and one was Annexin-1 by DNA sequencing. The other two clones were not identical to any of the genes submitted to GenBank (fig 1C). Of these, we further examined for Reg $1 \alpha$ and Annexin-1, functions of which are reported, and for GW112, which is the dominant species in DP3.

LEVELS OF REG 1 $\alpha$, ANNEXIN-1, AND GW112 mRNA IN COLONIC EPITHELIUM OF IBD, NON-IBD, AND CONTROL SUBJECTS

To examine if Reg $1 \alpha$, Annexin-1, and GW112 are preferentially expressed in active UC colonic epithelium, expression of these genes was further examined by real time PCR. In these assays, the relative amounts of gene transcripts were quantified in colonic epithelia of control subjects $(n=6)$, active UC $(n=14)$, 

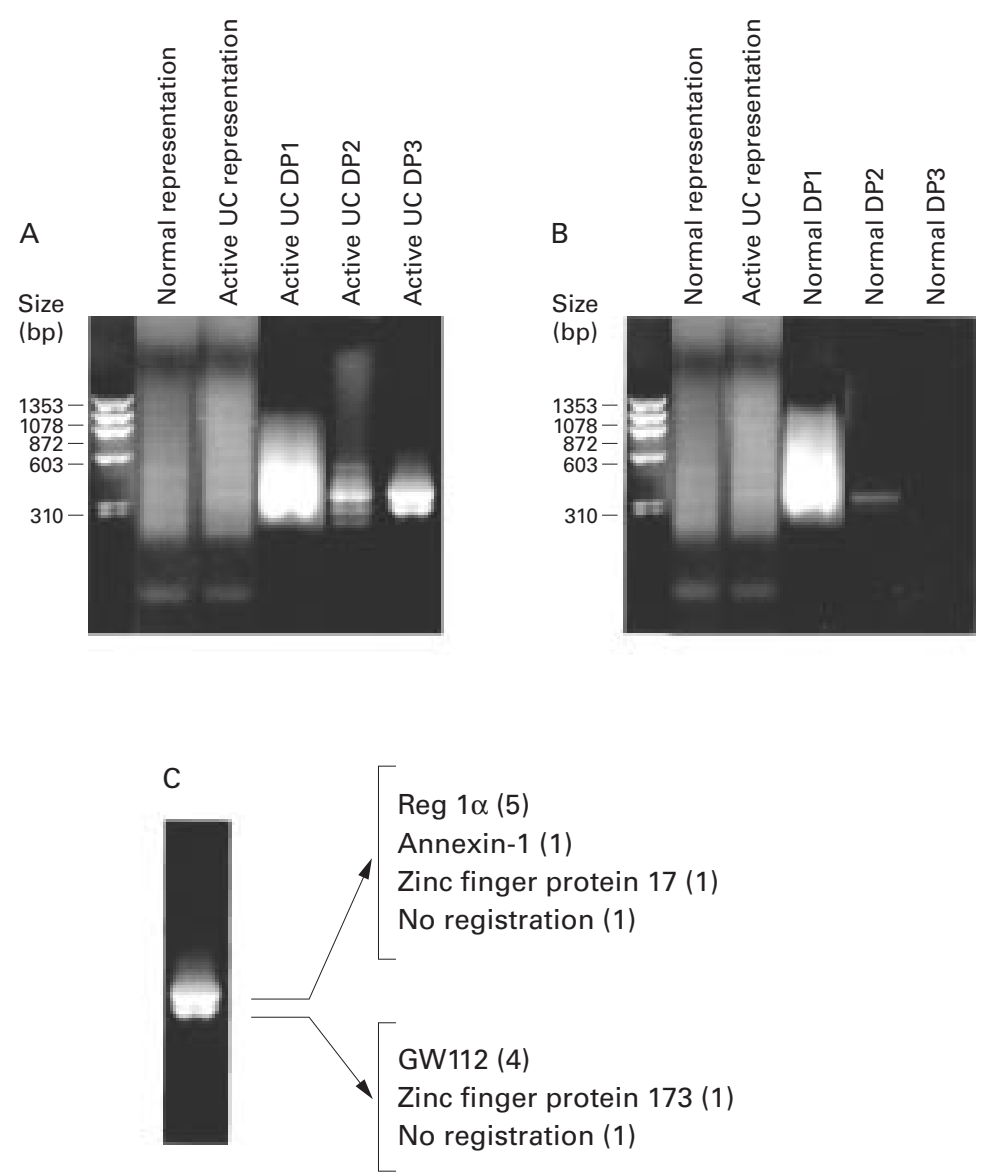

Figure 1 cDNA representation difference analysis ( $R D A$ ) of gene expression in normal and active ulcerative colitis (UC) colonic epithelia. (A) cDNA was derived from normal and active UC colonic epithelial cells, and used for $R D A$ with active UC representation as the "tester" and normal representation as the "driver." The original representations and the first (DP1), second (DP2), and third (DP3) difference products are shown. Note the presence of two discrete bands with no polydisperse DNAs in DP3. (B) cDNA was derived from normal and active UC colonic epithelial cells and used for RDA with normal representation as the "tester" and active UC representation as the "driver." The original representations and the first (DP1), second (DP2), and third (DP3) difference products are shown. Note the lack of discrete bands in DP3. (C) Identification of active UC DP3 by $D N A$ sequencing. Active UC DP3 was randomly cloned. Of 14 clones examined, five were found to be identical to Reg 1a, four were GW112, one was zinc finger protein 17, one was zinc finger protein 173, and one was Annexin-1 by DNA sequencing. The other two clones were not identical to any of the genes in the GenBank. Numbers in parentheses represent the number of identified clones. The GenBank accession numbers of Reg 1a, GW112, zinc finger proteins 17 and 173, and Annexin-1 are M18963, AF097021, NM-000700, $A A 205091$, and NM-003449, respectively.

inactive UC $(n=10)$, active Crohn's disease $(n=4)$, inactive Crohn's disease $(n=5)$, and non-IBD patients (ischaemic colitis $\mathrm{n}=5$, amoebic colitis $n=1$ ) using GAPDH gene transcripts as an internal control for standardisation (fig 2). The mean $\mathrm{C}_{\mathrm{T}}$ values of $\mathrm{GAPDH}$ gene transcripts of the colonic epithelium for each group were 26.0 (1.1) in normal, 24.6 (2.3) in active UC, 25.3 (1.9) in inactive UC, 25.4 (0.8) in active Crohn's, 24.8 (1.7) in inactive Crohn's, and 24.9 (1.2) in non-IBD. There were no significant differences in $\mathrm{C}_{\mathrm{T}}$ values of GAPDH gene transcripts among groups. The relative amounts of Reg $1 \alpha$ gene transcripts [fg/GAPDH $\left.\left(\mathrm{C}_{\mathrm{T}}=25.6\right)\right]$ of colonic epithelium in each of the above groups were: not detectable, 112.6 (44.1), 9.9 (8.0), 384.0 (366.5), $0.4(0.4)$, and 26.7 (15.0), respectively (fig 2A). Statistical analysis showed that the relative amount of Reg $1 \alpha$ gene transcripts in active UC colonic epithelium was significantly higher than in inactive UC and normal samples (active UC $v$ inactive UC, $\mathrm{p}<0.001$; active UC $v$ normal, $\mathrm{p}<0.001)$. We also detected significant amounts of Reg $1 \alpha$ gene transcripts in active Crohn's and in non-IBD active lesions. In inactive Crohn's disease, we detected small amounts (2.0) of Reg $1 \alpha$ gene transcripts only in one specimen. These results confirmed upregulation of Reg $1 \alpha$ gene expression in the colonic epithelium of inflamed colonic mucosa, including active UC, active Crohn's, and non-IBD active lesions. The relative amounts of GW112 gene transcripts in colonic epithelium in each group were 13.8 (2.9) in active UC, 5.0 (2.3) in inactive UC, 2.5 (2.2) in active Crohn's, 8.8 (5.7) in inactive Crohn's, and 3.2 (2.4) in non-IBD. In normal tissues, GW112 gene transcripts were not detectable (fig 2B). The relative amount of GW112 gene transcripts in active UC colonic epithelium was significantly higher than in inactive UC and normal specimens (active UC $v$ inactive UC, $\mathrm{p}<0.05$; active UC $v$ normal, $\mathrm{p}<0.001)$. We detected GW112 gene transcripts in two of four active Crohn's and in two of five inactive Crohn's and in three of six non-IBD active lesions. However, only in active UC was upregulation of GW112 gene expression confirmed to be statistically significant. We detected small amounts (1.0-7.0) of Annexin-1 gene transcripts in only five of 14 active UC and one of 10 inactive UC samples. Annexin-1 gene transcripts were not detectable in normal, active Crohn's disease, inactive Crohn's disease, or non-IBD (fig 2C).

DETECTION OF REG $1 \alpha$ AND GW112 GENE EXPRESSION IN ACTIVE UC COLONIC MUCOSA BY IN SITU HYBRIDISATION

The results of real time PCR suggested that expression of Reg $1 \alpha$ and GW112 genes was upregulated in the epithelium of inflamed colonic mucosa in active UC. To evaluate the pathological implications of Reg $1 \alpha$ and GW112 gene expression in inflamed colonic mucosa, it is necessary to determine the cellular basis of expression of these genes. In the next step, we examined localisation of Reg $1 \alpha$ and GW112 gene expression in the inflamed colonic mucosa in UC by ISH (fig 3). Paraffin embedded sections were hybridised to Dig labelled sense and antisense RNA probes complementary to Reg $1 \alpha$ or GW112 template DNA. Hybridisation of sections with Dig labelled antisense RNA probes of these genes showed that Reg $1 \alpha$ (fig 3A) and GW112 (fig 3B) signals were confined to the crypt epithelial cells whereas epithelial cells on the luminal surface were almost free of expression of these genes. Furthermore, expression of these genes was almost absent in mesenchymal cells. Hybridisation of serial sections with Dig labelled sense RNA probes of these genes showed sparse and weak background labelling of cells. Thus ISH confirmed the specific expression of Reg $1 \alpha$ and GW112 in crypt epithelial cells in active UC colonic mucosa. In normal colonic mucosa, Reg $1 \alpha$ or GW 112 gene expression was not detected by ISH (data not shown). 


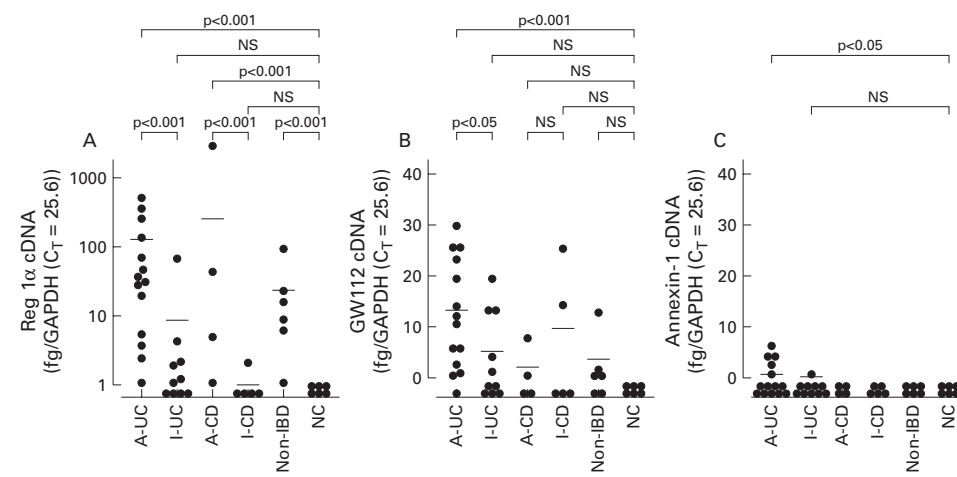

Figure 2 Relative quantitation of (A) Reg 1a, (B) GW112, and (C) Annexin-1 gene transcripts in inflammatory bowel disease (IBD), non-IBD, and normal colonic epithelia by real time polymerase chain reaction (PCR) assay. Total $R N A$ was extracted from colonic epithelia isolated from active ulcerative colitis (A-UC), inactive UC (I-UC), active

Crohn's disease (A-CD), inactive Crohn's disease (I-CD), non-IBD, and normal controls (NC) using glyceraldehyde-3-phosphate dehydrogenase (GAPDH) gene transcripts as internal control for standardisation. Each sample was tested in duplicate and the average of the two values was used for calculation. The difference between the two values was close to zero in all samples. After standardisation, the relative amounts of gene transcripts were expressed as the amount $(\mathrm{fg})$ per $G A P D H\left(C_{T}=25.6\right)$. Horizontal bars represent the mean value of each group.
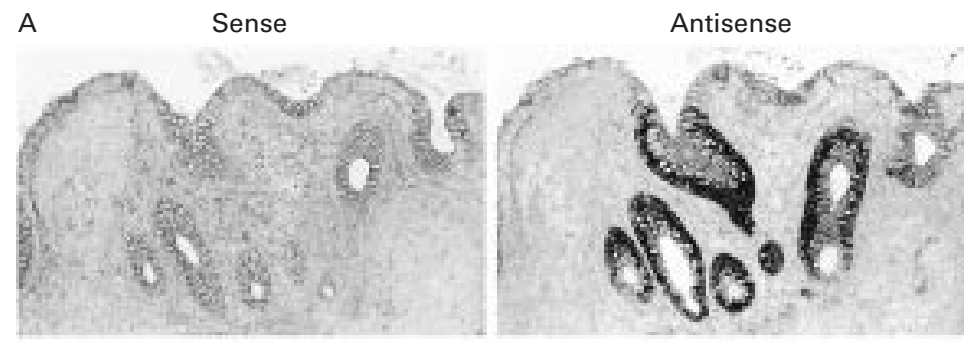

B Sense
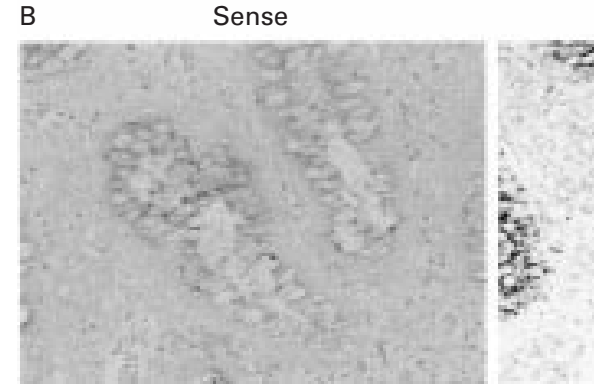

Antisense

Figure 3 In situ hybridisation of Reg $1 a$ and GW112 mRNAs in active UC colonic mucosa. Paraffin embedded sections were hybridised to digoxigenin (Dig) labelled sense and antisense RNA probes complementary to Reg 1a or GW112 template DNA. Hybridisation of sections with Dig labelled antisense RNA probes of these genes showed that $(A) \operatorname{Reg} 1 \alpha$ and (B) GW112 signals were confined to the crypt epithelial cells. Hybridisation of serial sections with Dig labelled sense RNA probes of these genes resulted in sparse and weak background labelling of cells. Original magnification $\times 25(A)$ and $\times 50$ (B).

COMPARISON OF SEQUENCES OF REG $1 \alpha$ cDNA DERIVED FROM IBD AND NON-IBD COLONIC

EPITHELIA

To further evaluate the pathological implications of Reg $1 \alpha$ gene expression in UC, we compared the sequences of Reg $1 \alpha$ cDNA derived from inflamed colonic epithelium of UC with that from ischaemic colitis. The 500 bp full length cDNA sequence of each four clones derived from colonic epithelium of active UC $(n=2)$ and ischaemic colitis $(n=2)$ was determined. In all clones examined, the Reg $1 \alpha$ cDNA sequences were not different
Reg $1 \alpha$ cDNA (fg/GAPDH $\left(\mathrm{C}_{\mathrm{T}}=15.5\right)$ )

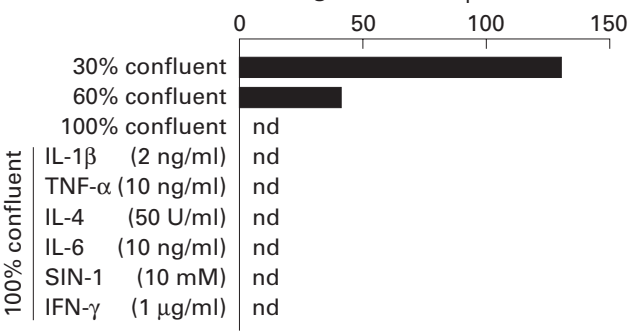

Figure 4 Effects of cell confluence and inflammatory cytokines on Reg 1a gene expression in a colonic epithelial cell line (HT29). Cells were harvested under 30\%, 60\%, and $100 \%$ confluent cell culture conditions and total RNA was extracted followed by real time polymerase chain reaction (PCR) assay to detect Reg 1 a transcripts. The effects of various cytokines and nitrous oxide on Reg 10 expression in HT29 were examined under 100\% confluent cell culture conditions. One day after HT29 cells became confluent, cells were cultured for 24 hours without or with

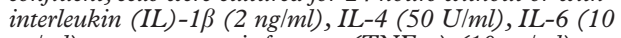
$\mathrm{ng} / \mathrm{ml})$, tumour necrosis factor a (TNF-a) $(10 \mathrm{ng} / \mathrm{ml})$, interferon $\gamma(I F N-\gamma)(1 \mu \mathrm{g} / \mathrm{ml})$, or

3-morpholinosydnonimine (SIN-1) (10 mM) in $2 \mathrm{ml}$ of RPMI1640 containing $10 \%$ fetal calf serum. Cells were harvested and total RNA was extracted followed by real time PCR to detect Reg 1a transcripts. Each sample was tested in duplicate, and the average of the two values was used for calculation. The difference between the two values was almost zero in all samples. After standardisation, the relative amount of gene transcripts was expressed as the amount $(\mathrm{fg})$ per GAPDH $\left(C_{T}=15.5\right)$. Results are from one of two experiments with identical results. nd, not detectable.

and were identical to wild-type Reg $1 \alpha$ (GenBank accession number M18963) in regenerating pancreatic islet cells. ${ }^{25}$

EFFECTS OF CELL CONFLUENCE AND

INFLAMMATORY CYTOKINES ON REG $1 \alpha$ GENE EXPRESSION IN COLONIC EPITHELIAL CELL LINE (HT29)

Finally, we examined whether upregulation of Reg $1 \alpha$ gene expression in inflamed colonic epithelium was mediated by inflammatory mediators. Recent studies have shown that Reg $1 \alpha$ expression in HT29 was associated with the cell growth period. ${ }^{26}$ We first examined if the level of Reg $1 \alpha$ gene expression depends on cell confluence by real time PCR. Increased confluence of these cells was associated with further downregulation of Reg $1 \alpha$ gene expression. Although at $30 \%$ and $60 \%$ cell confluence we detected significant amounts of Reg $1 \alpha$ gene transcripts (130 and $41 \mathrm{fg}$ Reg $1 \alpha$ transcripts/ GAPDH, respectively), at $100 \%$ cell confluence Reg $1 \alpha$ gene expression was not detectable (fig 4).

It was difficult to evaluate the effects of inflammatory cytokines on Reg $1 \alpha$ gene expression because the level of expression was associated with cell confluence. For this reason we examined the effects of various cytokines and nitrous oxide on Reg $1 \alpha$ expression in HT29 under $100 \%$ confluent cell culture conditions. One day after HT29 cells become confluent, cells were cultured without or with IL-1 $\beta(0.5,2$, or $10 \mathrm{ng} / \mathrm{ml}), \mathrm{IL}-4(5,50$, or 500 $\mathrm{U} / \mathrm{ml})$, IL-6 $(1,10$, or $100 \mathrm{ng} / \mathrm{ml})$, TNF- $\alpha$ $(1,10$, or $100 \mathrm{ng} / \mathrm{ml}), \operatorname{IFN}-\gamma(0.1,1$, or 10 $\mu \mathrm{g} / \mathrm{ml})$, or SIN-1 $(1,10$, or $100 \mathrm{mM})$ in $2 \mathrm{ml}$ of RPMI 1640 containing $10 \%$ fetal calf serum for 12,24 , or 48 hours. Cells were harvested and 
total RNA was extracted at 12,24 , and 48 hours of culture followed by real time PCR assay to detect Reg $1 \alpha$ transcripts. We could not detect Reg $1 \alpha$ gene expression under any condition or culture period studied at $100 \%$ confluency (fig 4 and data not shown).

\section{Discussion}

In the present study we found that Reg $1 \alpha$ and GW112 were the dominant species present in the RDA cDNA difference products with the active UC representation as "tester" and an excess amount of the normal representation as "drivers" (fig 1). cDNAs derived from normal and active UC colonic epithelium were digested with Dpn II and used as representations of normal and active UC colonic epithelium, respectively. It is possible that Dpn II fragments longer than several kilobase pairs may not be the RDA cDNA difference products, because of the low efficiency of PCR amplification of longer Dpn II cDNA fragments. For this reason, we may be able to find another predominant gene species preferentially expressed in colonic epithelium of active UC by using other four base pair recognition restriction enzymes than Dpn II to generate representations. Of note, cDNA RDA cannot be used negatively to prove something is not different for the same reason. Thus the RDA result with the normal representation as "tester" and an excess amount of the active UC representation as "drivers" (fig 1B) in which no discrete bands were detected does not mean that predominant gene species were not preferentially expressed in normal colonic epithelium.

Reg $1 \alpha$ was discovered as a gene upregulated in regenerating pancreatic islet cells. ${ }^{25}{ }^{27}$ Subsequent studies reported that Reg protein was identical to pancreatic stone protein which had been found in stones of patients with chronic calcifying pancreatitis. ${ }^{28}$ The sequence of the human Reg $1 \alpha$ gene predicts a 166 amino acid peptide with an $\mathrm{N}$ terminal signal peptide of 23 amino acids. ${ }^{25}$ The recombinant rat Reg protein is reported to be mitogenic to isolated rat islet cells, ${ }^{29} \beta$ cell line, ${ }^{30}$ and pancreatic ductal cells. ${ }^{31}$ Recently, ectopic expression of the Reg $1 \alpha$ gene has been reported outside the pancreas, including the enterochromaffin-like (ECL) cells of the rat gastric corpus, Paneth cells, and columnar cells of human duodenum $^{32}$ and human colorectal cancer. ${ }^{33}$

In the present study, we found for the first time that Reg $1 \alpha$ gene expression was upregulated in the epithelium of inflamed colonic mucosa in IBD and non-IBD (fig 2A). Reg $1 \alpha$ gene expression was negative in normal colonic epithelium as already reported at mRNA and protein levels. ${ }^{25}{ }^{34} \mathrm{We}$ also found that Reg $1 \alpha$ gene expression was confined to crypt epithelial cells whereas epithelial cells on the luminal surface were almost free of Reg $1 \alpha$ gene expression (fig 3A). The fact that upregulation of Reg $1 \alpha$ gene expression in the epithelium of inflamed colonic mucosa is common in IBD and non-IBD strongly suggests the important physiological roles of Reg $1 \alpha$ protein.

Previous studies indicated that the protein of Reg family, pancreatitis associated protein 1, confers pancreatic cell line resistance to apoptosis. $^{35}$ In the colon, antiapoptotic $\mathrm{Bcl}-2$ immunostaining is prominent in the crypt epithelium and epithelial cells on the luminal surface are almost free of Bcl-2 staining. ${ }^{20}{ }^{36}$ The identical distribution of Bcl-2 and Reg $1 \alpha$ in the colonic epithelium suggests that Reg $1 \alpha$ might exert antiapoptotic effects in the inflamed colonic epithelium.

Reg $1 \alpha$ is reported to be a growth factor for gastric mucous cells ${ }^{37}$ and thus may also be a growth factor for colonic epithelial cells. Recently, a receptor for Reg $1 \alpha$ was identified, ${ }^{30}$ which is encoded by the exostoses-like gene and mediates the growth signal of Reg $1 \alpha$ for $\beta$ cell regeneration. If Reg $1 \alpha$ mediates the growth of colonic epithelial cells, it becomes important to examine expression of Reg $1 \alpha$ receptor (Reg $1 \alpha \mathrm{R}$ ) on colonic epithelial cells, because deficient expression of Reg $1 \alpha \mathrm{R}$ or altered Reg $1 \alpha \mathrm{R}$ signal in IBD colonic epithelial cells might be one of the causative mechanisms of disturbed reconstitution of IBD colonic mucosa. Further studies are required to clarify this point.

In the rat gastric mucosa, Reg $1 \alpha$ gene expression is confined to gastric ECL cells. Gastrin stimulates the production of Reg protein in gastric ECL cells and regulates gastric Reg production. ${ }^{37}$ In inflamed colonic mucosa, regulation of Reg $1 \alpha$ gene expression is probably different from that of gastric Reg $1 \alpha$ gene expression as the inflamed colonic mucosa crypt epithelial cells, including goblet cells not ECL cells, expressed the Reg $1 \alpha$ gene (fig 3A). Results of our in vitro study using colonic epithelial cell line HT29 suggested that cell confluence rather than inflammatory mediators regulate $\operatorname{Reg} 1 \alpha$ gene expression (fig 4). ${ }^{26}$ Thus altered cell-cell contact in the epithelium of inflamed colonic mucosa may regulate Reg $1 \alpha$ gene expression, and Reg $1 \alpha$ expression in the colonic epithelium may be a marker of altered cell-cell contact. In five of 10 inactive UC and in two of five inactive Crohn's disease samples, we detected Reg $1 \alpha$ gene expression. This finding suggests that cell-cell contact and epithelial reconstitution would still be incomplete in these Reg $1 \alpha$ positive epithelia although these appeared to be almost intact endoscopically and histologically, implying that this might be a marker of relapse. Further examination using a large population is necessary to confirm this conclusion. In this context, it should be stressed that we cannot categorically exclude the possible effect of inflammatory mediators on Reg $1 \alpha$ gene expression because treatment of confluent "transformed" HT29 cells with inflammatory mediators may be an unsuitable model to examine the effect of these mediators on Reg $1 \alpha$ gene expression of "non-transformed" colonic epithelial cells in the inflamed colonic mucosa.

It is well known that colonic cancer develops from inflamed colonic mucosa in UC. Recent studies reported that mutations of Reg $1 \alpha$ that prevent secretion are associated with gastric ECL cell carcinoids ${ }^{38}$ and that Reg $1 \alpha$ expression may be a sensitive marker of colorectal 
mucosa at risk for the development of neoplasia. ${ }^{34}$ However, the mere presence of Reg $1 \alpha$ protein in the colonic epithelium cannot be a marker for the development of colonic cancer because Reg $1 \alpha$ expression was detected in non-IBD colonic epithelium, which does not develop neoplasias (fig 2A). We then examined if there exists mutations of Reg $1 \alpha$ in the colonic epithelium in UC and whether they could be the causative factor of colonic cancer. To address this issue, we compared the sequences of Reg $1 \alpha$ cDNA derived from inflamed colonic epithelium of UC and ischaemic colitis, which heals spontaneously and does not progress to colonic cancer. In all clones examined, Reg $1 \alpha$ cDNA sequences were not different and were identical to wild-type Reg $1 \alpha$ in regenerating pancreatic islet cells. ${ }^{25}$ Thus it is unlikely that Reg $1 \alpha$ is involved in colonic carcinogenesis in UC, although the number of subjects examined in this study was limited.

GW112 is a gene cloned from human myeloblasts. To our knowledge, no study has investigated GW112 distribution, and the function of GW112 is unknown. Preferential expression of GW112 in the crypt epithelium in inflamed colonic mucosa in UC (figs $2 \mathrm{~B}, 3 \mathrm{~B}$ ) suggests an important physiological role of GW112, although further studies are necessary to determine the exact role.

In summary, using RDA, we identified seven candidate genes that are probably upregulated in active UC colonic epithelium. Of these, Reg $1 \alpha$ and GW 112 were found to be the dominant species in inflamed colonic epithelium and their expression was confined to the crypt epithelium, as demonstrated by ISH. Reg $1 \alpha$ expression in the colonic epithelium could be considered as a marker of altered cell-cell contact and may be related to regeneration of colonic epithelia in inflamed colonic mucosa, similar to its role in pancreatic islets. Gene targeting studies to elucidate the in vivo function of Reg $1 \alpha$ are currently in progress in our laboratory. Further studies are necessary to clarify the biological function of Reg $1 \alpha$ in the inflamed colonic epithelia.

This work was supported in part by Sato Memorial Cancer Research Fund and Grant-in-Aid for Scientific Research (C).

1 Owen DA, Kelly JK. Inflammatory diseases of the gastrointestinal tract. Mod Pathol 1995;8:97-108.

2 Podolsky DK. Mucosal immunity and inflammation V. Innate mechanisms of mucosal defense and repair; the best offense is a good defense. Am F Physiol 1999;277:G495-9.

3 Sadlack B, Merz H, Schorle H, et al. Ulcerative colitis-like disease in mice with a disrupted interleukin-2 gene. Cell 1993;75:253-61.

4 Kuhn R, Lohler J, Rennick D, et al. Interleukin-10-deficientmice develop chronic enterocolitis. Cell 1993;75:263-74.

5 Mombaerts P, Mizoguchi E, Grusby MJ, et al. Spontaneous development of inflammatory bowel disease in T cell receptor mutant mice. Cell 1993;75:275-82.

6 Hermiston ML, Gordon JI. Inflammatory bowel disease and adenomas in mice expressing a dominant negative N-cadherin. Science 1995;270:1203-7.

7 Katz KD, Hollander CM, Vadheim CM, et al. Intestinal permeability in patients with Crohn's disease and thei healthy relatives. Gastroenterology 1989;97:927-31.

8 Serafini EP, Kirk AP, Chambers TJ. Rate and pattern of epithelial cell proliferation in ulcerative colitis. Gut 1981;22 648-52.

9 Toy LS, Yio XY, Lin A, et al. Defective expression of gp180, a noble CD8 ligand on intestinal epithelial cells, in inflammatory bowel disease. $\mathcal{F}$ Clin Invest 1997;100:2062-71.
10 Nakazawa A, Watanabe M, Kanai T, et al. Functional expression of costimulatory molecule CD86 on epithelial cells in the inflamed colonic mucosa. Gastroenterology 1999;117:536-45.

11 Panja A, Goldberg S, Eckmann L, et al. The regulation and functional consequence of proinflammatory cytokine binding on human intestinal epithelial cells. F Immunol 1998;161:3675-84.

12 Woywodt A, Neustock P, Kruse A, et al. Cytokine expression in intestinal mucosal biopsies. In situ hybridisation of the mRNA for interleukin-1 beta, interleukin-6 and tumor necrosis factor-alpha in inflammatory bowel disease. Eur Cytokine Netw 1994;5:387-95.

13 Gibson P, Rosella O. Interleukin 8 secretion by colonic crypt cells in vitro: response to injury suppressed by butyrate and enhanced in inflammatory bowel disease. Gut 1995;37: 536-43.

14 Z'Graggen K, Walz A, Mazzucchelli L, et al. The C-X-C chemokine ENA-78 is preferentially expressed in intestinal epithelium in inflammatory bowel disease. Gastroenterology 1997;113:808-16.

15 Sakai T, Kusugami K, Nishimura H, et al. Interleukin 15 activity in the rectal mucosa of inflammatory bowel disease. Gastroenterology 1998;114:1237-43.

16 Kolios G, Rooney N, Murphy CT, et al. Expression of inducible nitric oxide synthase activity in human colon epithelial cells: modulation by $\mathrm{T}$ lymphocyte derived cytokines. Gut 1998;43:56-63.

17 Garland CF, Lilienfeld AM, Mendeloff AI, et al. Incidence rates of ulcerative colitis and Crohn's disease in 15 areas of the United States. Gastroenterology 1981;81:1115-24.

18 Scowcroft CW, Sanowski RA, Kozarek RA. Colonoscopy in ischemic colitis. Gastrointest Endoscopy 1981;27:156-61.

19 Matts F. The value of rectal biopsy in the diagnosis of ulcerative colitis. Qf Med 1961;30:393-407.

20 Iimura $M$, Nakamura $T$, Shinozaki $S$, et al. Bax is downregulated in inflamed colonic mucosa of ulcerative colitis Gut 2000;47:228-35.

21 Hubank M, Schatz D. Identifying difference in mRNA expression by representation difference analysis of cDNA. Nucleic Acids Res 1994;22:5640-8.

22 Chomczynski P, Sacchi N. Single-step method of RNA isolation by acid guanidinium thiocyanate-phenol-chloroform extraction. Anal Biochem 1987;162:156-9.

23 Kimura H, Morita M, Yabuta Y, et al. Quantitative analysis of Epstein-Barr virus load by using a real-time PCR assay. f Clin Microbiol 1999;37:132-6.

24 Heid CA, Stevens J, Livak KJ, et al. Real time quantitative PCR. Genome Res 1996;6:986-94.

25 Watanabe T, Yonekura H, Terazono K, et al. Complete nucleotide sequence of human reg gene and its expression in normal and tumoral tissues. F Biol Chem 1990;265: $7432-9$

26 Bernard-Perrone FR, Renaud WP, Guy-Crotte OM, et al. Expression of reg protein during cell growth and differentiation of two human colon carcinoma cell lines. $\mathcal{F}$ Histochem Cytochem 1999;47:863-70.

27 Terazono K, Yamamoto $\mathrm{H}$, Takasawa $\mathrm{S}$, et al. A noble gene activated in regenerating islets. F Biol Chem 1988;263: 2111-14

28 Rouquier S, Verdier JM, Iovanna J, et al. Rat pancreatic stone protein messenger RNA. F Biol Chem 1991;266:78691.

29 Watanabe T, Yonemura Y, Yonekura H, et al. Pancreatic beta-cell replication and amelioration of surgical diabetes by reg protein. Proc Natl Acad Sci USA 1994;91:3589-92.

30 Kobayashi S, Akiyama T, Nata K, et al. Identification of a receptor for Reg (regenerating gene) protein, a pancreatic B-cell regeneration factor. F Biol Chem 2000;275:10723-6.

31 Zenilman ME, Chen J, Magnuson TH. Effect of reg protein on rat pancreatic ductal cells. Pancreas 1998;17:256-61.

32 Senegas-Balas FO, Figarella CG, Amouric MA, et al. Immunocytochemical demonstration of a pancreatic secretory protein of unknown function in human duodenum. $\mathcal{F}$ Histochem Cytochem 1991;39:915-19.

33 Rechreche $\mathrm{H}$, Montalto G, Mallo GV, et al. pap, reg $1 \alpha$ and reg $1 \beta$ mRNAs are concomitantly upregulated during
human colorectal carcinogenesis. Int $\mathscr{f}$ Cancer $1999 ; 81$ : human

34 Zenilman ME, Kim S, Levine BA, et al. Ectopic expression of reg protein: a marker of colorectal mucosa at risk for neoplasia. F Gastrointest Surg 1997;1:194-202.

35 Ortiz EM, Dusetti NJ, Vasseur S, et al. The pancreatitisassociated protein is induced by free radicals in AR4-2J cells and confers cell resistance to apoptosis. Gastroenterology 1998;114:808-16.

36 Krajewski S, Krajewski M, Shabaik A, et al. Immunohistochemical determination of in vivo distribution of Bax, a
dominant inhibitor of Bcl-2. Am $\mathcal{F}$ Pathol 1994;145:132336.

37 Fukui H, Kinoshita Y, Maekawa T, et al. Regenerating gene protein may mediate gastric mucosal proliferation induced by hypergastrinemia in rats. Gastroenterology 1998;115: 1483-93.

38 Higham AD, Bishop LA, Dimaline R, et al. Mutation of reg $1 \alpha$ are associated with enterochromaffin-like cell tumor development in patients with hypergastrinemia. Gastroenterology 1999;116:1310-18. 